\title{
Combination Between Surgical Excision and Pressure Therapy for Ear Keloids in a Pediatric Population: Clinical Results
}

\author{
Ibrahim Cherry, Tamara Sadighi, Diane Franck \\ Hopital Universitaire des Enfants Reine Fabiola, Université Libre de Bruxelles, Brussels, Belgium
}

Email address:

Ibrahim.cherry@hotmail.com (I. Cherry),ortho.sina@skynet.be (T. Sadighi)

\section{To cite this article:}

Ibrahim Cherry, Tamara Sadighi, Diane Franck. Combination Between Surgical Excision and Pressure Therapy for Ear Keloids in a Pediatric Population: Clinical Results. Journal of Surgery. Vol. 9, No. 3, 2021, pp. 103-108. doi: 10.11648/j.js.20210903.12

Received: April 11, 2021; Accepted: April 22, 2021; Published: May 8, 2021

\begin{abstract}
Introduction: A single effective therapeutic option for ear keloids has yet to be discovered. Many treatments have been studied with variable effectiveness, side effects and complications. Pressure therapy using custom molded clip is a noninvasive treatment with minimal side effects. In the present study, the recurrence rate of a treatment protocol combining excisional surgery and pressure therapy on ear keloids is being studied in a pediatric population. Materials and Methods: The charts of 22 consecutive patients treated between January 2008 and January 2019 were reviewed. All patients were under 18 years of age and clinically diagnosed with, at least, one keloid of the ear. They all underwent complete surgical excision with primary closure followed by early postoperative pressure therapy using custom molded clips. The collected data included patient age at time of diagnosis and surgery, sex, skin type, familial and personal history, etiology and anatomical location of the keloid, and recurrence. Patients or parents were interviewed by phone call and reviewed by an independent examiner to evaluate the current evolution of their scar (s). Results: 22 patients were included in the study, representing a total of 26 keloids of the ear. The male to female ratio was of 3:4. The mean age at diagnosis was 9.7 years old. The mean follow-up time was 5.2 years. Four ears needed a single corticoid injection in addition to the initial protocol. Follow-up observations showed a recurrence rate of $15.4 \%$. Conclusion: Excisional surgery combined with pressure therapy is an effective and well-tolerated treatment for ear keloids in a pediatric population. Although residual symptoms are sometimes observed, it seems to be better tolerated than other, more invasive, therapies.
\end{abstract}

Keywords: Ear Keloids, Pediatric, Surgical Excision, Pressure Therapy, Recurrence Rate

\section{Introduction}

\subsection{Wound Healing}

The skin is the barrier that protects internal tissues against trauma. After an initial traumatic event, starts the first stage of wound healing: the hemostatic phase. The scarring process is then subdivided into three overlapping steps: inflammation, cellular proliferation and extracellular matrix (ECM) remodeling [1]. When those steps work in harmony, wound healing results in a healthy and mature scar. If the scarring process is perturbed, a hypertrophic or keloid scar can occur. Unlike hypertrophic scars (HTS), keloids extend beyond the area of injury [2]. Clinical differentiation between both scar types is intricate (Annex II). A keloid starts as an unaesthetic amorphous nodule that can grow large enough to cause pruritus, pain, secondary infections, mobility restriction and loss of self-esteem [3, 4]. In order to support clinical diagnosis, histopathologic features have been described as pathognomonic to keloid specimens [5].

\subsection{Physiopathology of Keloids}

The first challenge in keloid management resides in its incomplete understood physiopathology. A detailed explanation of the process is beyond the scope of this article. Keloids pathogenesis can be summarized as an imbalance between collagen anabolism and catabolism that involves both environmental and genetic factors [6]. Dark skin phenotype is well-established risk factors for keloid genesis. Authors have observed a ratio of keloids incidence going up 
to $15: 1$ between darker and lighter skin color [7].

Keloids can occur all over the body, but the incidence is higher in the ears, the back of the neck, the anterior chest, the deltoid region and the upper back. The ears, and mainly the lobes, are the most common anatomical site of keloids in the 10-20 age group [8]. Indeed, earrings in children are a common practice. They induce chronic tension and irritation leading to secretion of proinflammatory mediators and ultimately to pathological scarring [9].

\subsection{Avoiding Recurrence}

Recurrence is a common clinical feature of keloids. Many treatment modalities are described in the literature including surgical excision, intralesional steroid injections, radiation therapy, silicon and non-silicone based occlusive dressings, cryosurgery, laser treatment and antitumoral or immunosuppressive agents [10]. There is no universally accepted protocol for ear keloids management. The lack of knowledge about the physiopathology and the impossibility to get an appropriate animal model (keloids scars are unique to human) can explain the difficulty in finding an appropriate treatment $[11,12]$. As Niessen et al. explain "the less that is known about a disease, the more therapeutic modalities seem to be available" [13].

Avoiding recurrence in the auricular area is critical. Each recurrence allows the keloid to consume more healthy soft tissue and increases the difficulty of an acceptable surgical reconstruction [14]. Inefficiency of surgical excision alone has been proven. Recurrence rate ranges from $45 \%$ to $100 \%$ [12]. This rate decreases when an adjuvant therapy is combined. Postoperative radiotherapy and pressure therapy are both very successful adjuvant treatment, showing a recurrence rate of respectively $2-33 \%$ [15] and $10-28 \%$ [16, 17]. However, irradiation must be avoided in children because of its increased risk of radiation-induced carcinogenesis [15].

Since Nason introduced, in 1942, the efficacy of pressure therapy after surgical excision [18], multiple pressure devices have been introduced and reported promising results [16, 17, 19]

Even though the pediatric population has its own specificity, there are few demographic and therapeutic studies focusing on this patient group. No study has assessed the effectiveness of pressure therapy on ear keloids in children. In the present study, the use of a custom molded pressure device as an adjuvant therapy for ear keloids was evaluated in a pediatric population.

\section{Materials and Methods}

\subsection{Study Design}

This retrospective study was performed after agreement of the Ethics Committee of the Queen Fabiola Children's University Hospital.

The charts of 22 consecutive patients treated between January 2008 and January 2019 were reviewed. All patients were under 18 years of age and clinically diagnosed with, at least, one keloid of the ear. They all underwent complete surgical excision with primary closure followed by early postoperative pressure therapy using custom molded clip.

Collected data from the charts included patient's age at time of diagnosis and surgery, sex, skin type according to the Fitzpatrick scale, etiology and precise location of the ear keloid, familial and personal history of keloids, previous treatment and recurrence rate. Demographic and clinical variables were compared between the recurrent et nonrecurrent group to establish possible risk factors for recurrence.

After data collection, patients were interviewed by phone call. If patients were under 18 years old, contact was made through their parent or legal guardian. They were also reviewed by an independent examiner to evaluate the current evolution of their scar (s): clinical aspect (complete regression, residual elevation or recurrence) and residual symptom (s) (pruritus and/or tenderness).

The recurrence rate was defined as the primary outcome of the study.

\subsection{Treatment Protocol}

Keloid excision is performed as an ambulatory surgery. The excised lesion is sent to the pathologist for diagnosis confirmation. Non resorbable stiches are removed within 2 weeks. An appointment is then planned with the orthotist for clip manufacture. Pressure therapy starts three to four weeks after surgery. Patient must wear the clip for 23 hours a day until the end of the treatment. If the patient is treated for bilateral ear keloids, the device must be alternatively applied on each ear to minimize discomfort during sleep.

Patients are followed up at the outpatient clinic after one week of pressure therapy, and, afterwards, every 3 months. During scar examination, the device is temporarily removed. Recurrence is evaluated, side effects are documented, and comfort is discussed with the patient and the parents. If side effects are observed, pressure is adjusted or wearing time is reduced.

\subsection{Clip Manufacture}

The 3 layers pressure device applies continuous and homogeneous pressure on the operated ear. Its transparency allows for continuous examination of the scar and skin perfusion control (Figure 2).

The prosthetist in charge of manufacturing the clip was $\mathrm{T}$. Sadighi from Ortho Sina. This process is subdivided into 2 steps and takes around 1 week to be completed:

I. Ear molding: an impression of the operated ear is molded using vinyl polysiloxane (liquid silicone). Fuji Rock Plaster is then used to create a positive mold and dried in the oven for 8 hours at $100^{\circ}$ Celsius.

II. Layers of the clip: (a) Inner layer: a silicone sheet is applied on the dried plaster. Both components are placed in the oven for 8 hours at $50^{\circ}$ Celsius. This step allows the silicone to soften in order to take the shape of the ear. (b) Medial layer: a $3 \mathrm{~mm}$ thick plastic sheet is applied and vacuumed on the previously prepared set. (c) Outer layer: the 
previous step is repeated to form the outer layer of the clip. A screw system is added allowing for pressure adjustment.

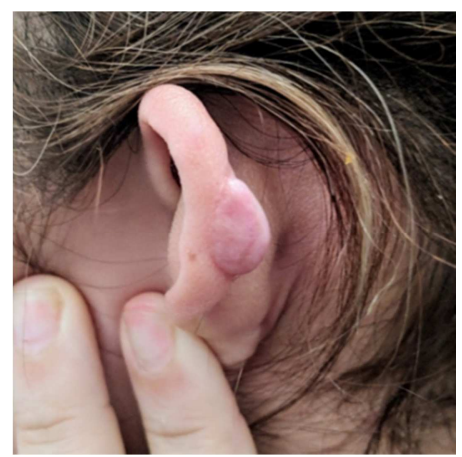

Figure 1. Keloid scar of the helix before any treatment.

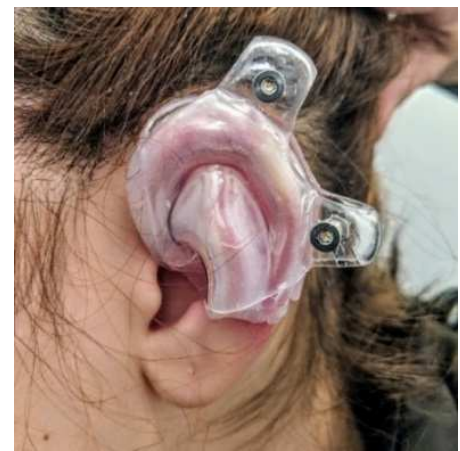

Figure 2. Pressure clip molded on the left helix after surgical excision of the keloid.

\section{Results}

\subsection{Demographic and Clinical Characteristics}

22 patients were included in the study, representing a total of 26 keloid scars of the ear. The male to female ratio was $3: 4$, with a mean age of 9.7 years (2-17 years) at time of diagnosis.

With respect to the site, 16 lesions were located on the right ear and 10 on the left. Four patients presented bilateral keloids. Location of the keloids was on the lobule (12 ears; 46.1\%), retro-auricular (9 ears; 34.6\%) and on the helix (5 ears; 19.2\%) The most frequent cause was piercing (14 ears; 53,8\%). Otoplasty was also a common etiology ( 9 ears; $34.6 \%$ ). The studied group included 1 case of scratch injury on chicken pox eruption and 2 cases of post-traumatic wound.

The number of primary keloids was 16 , and 10 had histories of treatment failure after excisional surgery performed in other institutions. None of the patients was previously treated with pressure therapy (Table 1).

\subsection{Follow-up Assessment}

All patients completed the protocol, with a total treatment period ranging from 8 to 18 months (mean: 10,8). Mean follow-up time after the end of the pressure therapy was 5.1 years $(1.2-9.7)$. Mean time between excisional surgery and pressure treatment was 25 days $(22-27)$.

All patients underwent phone call interviews and were seen for clinical examination by an independent examiner.
Twenty of them, representing 22 ears (84.6\%), did not show keloid recurrence. To achieve complete regression of the scar, four ears needed a single corticoid injection in addition to the initial protocol. In the non-recurrent group, residual symptoms were documented in 3 patients. Two ears presented pruritus and one showed tenderness.

The remaining two patients (4 ears; 15.4\%) showed recurrence on physical examination. Both patients had dark pigmented skins, bilateral and retro-auricular keloids scars. One of them repeated the treatment protocol and still showed recurrence.

Table 1. Demographic and clinical characteristic.

\begin{tabular}{llll}
\hline $\mathbf{N}=\mathbf{2 2}$ patients & \multicolumn{3}{c}{$\mathbf{N = 2 6}$ ears } \\
\hline Age (yrs) & $9.7(2-17)$ & $\begin{array}{l}\text { Previous } \\
\text { treatment }\end{array}$ & $10(38.5 \%)$ \\
Gender (male) & $9(40.9 \%)$ & Site of keloid & \\
Fitz-Patrick scale & & Lobule & $12(46.2 \%)$ \\
III & $8(36.3 \%)$ & Retro-auricular & $9(34.6 \%)$ \\
IV & $5(22.7 \%)$ & Helix & $5(19.2 \%)$ \\
V & $7(31.8 \%)$ & Etiology & \\
VI & $2(9.1 \%)$ & Piercing & $14(53.8 \%)$ \\
Family history & $7(31.8 \%)$ & Otoplasty & $9(34.6 \%)$ \\
Personal history & $4(18.2 \%)$ & Trauma & $3(11,5 \%)$ \\
Bilateral location & $4(18.2 \%)$ & &
\end{tabular}

One ear suffered from maceration during pressure treatment and needed clip adjustment. No other side effects were noted (Table 2).

Risk factors for recurrence

Differences between the recurrence and nonrecurrence group were identified in order to establish risk factor for treatment failure. Significant differences were observed in several parameters: retro-auricular and bilateral locations of the keloid (s), etiology of the keloid (piercing and otoplasty) and pressure therapy duration. No significant differences were observed in the remaining data (Table 3).

Table 2. Recurrence and side effects $(N=26$ ears).

\begin{tabular}{ll}
\hline Pressure therapy duration (months) & $\mathbf{8 - 1 8}(\mathbf{1 0 . 8 )}$ \\
\hline Side effects & 1 \\
Maceration & 0 \\
Infection & 0 \\
Necrosis & 4 \\
Recurrence & 1 \\
Residual symptoms (non-recurrent group) & \\
Tenderness & 2 \\
Pruritus & \\
\hline
\end{tabular}

Table 3. Demographic differences between the recurrence and nonrecurrence ears.

\begin{tabular}{lll}
\hline & $\begin{array}{l}\text { Recurrence } \\
\mathbf{N = 4}(\mathbf{1 5 . 4 \% )})\end{array}$ & $\begin{array}{l}\text { Non-recurrence } \\
\mathbf{N = 2 2}(\mathbf{8 4 . 6 \% )}\end{array}$ \\
\hline Site of lesion & & \\
Lobule & 0 & 12 \\
Retro-auricular & 4 & 5 \\
Helix & 0 & 5 \\
Initiating event & & \\
Piercing & 0 & 14 \\
Otoplasty & 4 & 5 \\
Trauma & 0 & 3 \\
Previous treatment & 2 & 8 \\
Pressure therapy duration & $14(13-15)$ & $10.2(8-18)$ \\
(months) & & \\
\hline
\end{tabular}




\section{Discussion}

The literature on keloid management describes many different treatment protocols. Most of them are combining surgery with intralesional corticosteroids, radiation therapy or pressure therapy. Corticosteroids injection is commonly used in keloid prevention and is the second most used adjuvant therapy after compression garments [20]. Both its accessible cost and ease to administer justify its wide use. However, the concentration of the drug is not standardized, and repeated injections are often necessary to reach satisfying results. Side effects of corticosteroids injections include pain upon injection, pigmentary alterations, dermal atrophy [14, 21].

Low-dose radiation as an adjuvant treatment has shown promising results in reducing keloid recurrence. Success rates varies between 67 and 98\% [15]. A review showed that radiation therapy meets high rates of patient compliance. However, it also showed that side effects can sometimes be severe.

Reported cases of malignant tumor after keloid radiation have limited its use, especially on a pediatric population [15].

Pressure therapy "against the deformity of scars" was first described in the sixteenth century by Ambroise Paré [22]. More recently, authors have investigated the underlying mechanism of action. Pressure clips inhibit the scar growth by keeping it in a limited space. Moreover, the compression applied on the capillaries decreases oxygen supply to the tissue, causing fibroblast apoptosis. This hypoxia maintains collagen disintegration since it is an oxygen-independent process $[23,24]$. Pressure therapy is a non-invasive treatment with minimal side effects. The description of possible adverse effects includes maceration, eczema, smell or skin necrosis if the applied pressure is too high [21].

\subsection{Ear-clip Benefits}

The use of a silicone sheet ensures hydration of the corneum by limiting water evaporation. This water retention reduces capillary activity, hyperemia and symptoms like pruritus and hyperpigmentation. Scar dehydration also causes electrostatic changes that promote collagen deposition [24, 25]. Additionally, Choi et al. observed a decreased expression of TGF- $\beta 1$ in keloid specimens treated with silicone gel [2]. Those combined features maintain a great environment for healthy scarring.

A well-controlled compression on the scar is crucial to avoid harmful complications. Too high pressure may lead to ischemia, and too low pressure has no beneficial effect on preventing keloid recurrence. To exceed capillary tension, the exerted pressure should be at least $24 \mathrm{mmHg}$, but must stay below $30 \mathrm{mmHg}$ [27]. The studied device applies continuous and homogeneous compression on the targeted area, enough to induce a hypoxia in the keloidal tissue, yet not enough to lead to necrosis nor infection to the surrounding tissues. Additionally, if the pressure initially applied needs correction, the third layer of the device allows for adjustment in the amount of pressure. In the reported cases, one patient developed maceration and needed pressure correction. None suffered from ischemic complications.

Table 4. Studies assessing the effectiveness of different pressure devices (ranked by mean follow-up period).

\begin{tabular}{llll}
\hline Authors & N. of ear keloids & Mean follow-up & Recurrence rate \\
\hline Park et al. [17] & 883 & 18 months & $10,6 \%$ \\
Kadouch et al. [16] & 23 & 23 months & $17 \%$ \\
Present study & 26 & 5.1 years $(1.2-9.7)$ & $15.4 \%$ \\
Tanaydin et al. [19] & 88 & 6.5 years $(0.9-12.3)$ & $29.5 \%$ \\
\hline
\end{tabular}

The list of features for the ideal pressure device advanced by Chalian et al. mentions that it must be lightweight, aesthetically acceptable, affordable, easily placed and removed by the patient and applying a uniform pressure. It should also be comfortable to get the best compliance, and transparent allowing continuous control of perfusion [28]. The studied pressure clip fulfills most criterias. Unfortunately, the device meets financial limitation. One child could not benefit from this treatment due to its cost. Despite its proven efficiency against keloids [16, 17, 19], compression garments are not refund by Belgian mutual insurances, except for burn patients.

\subsection{Importance of Follow-up}

Tanaydin et al. observed that ear keloids can recur during the 3 years following the end of the pressure therapy [19]. When patients are satisfied with the apparent result, it is common to see them discontinuing their follow-up at the outpatient clinic. Previous studies have assessed the effectiveness of different pressure devices and observed high success rates $[16,17]$. However, patients were followed for relatively short periods (mean $<2$ years) which may have a biased interpretation of their results. In our study, all patients but one have a follow-up period exceeding 3 years. This long-term observance allowed for proper evaluation of keloid recurrence (Table 4).

\subsection{Risk Factors for Recurrence}

Considering the small population size of our study, establishing strong conclusions was not possible. However, our results have illustrated some tendencies:

(1) To reduce discomfort, patients treated for bilateral keloids were instructed to wear the device on one ear each day and to alternate. Pressure was then applied on the scar for nearly half the conventional amount of time. This reduced compression may have constituted a potential risk factor of keloid recurrence. Indeed, observations on both recurrent patients $(100 \%)$ showed bilateral location of the keloids. Whereas, only $12 \%$ presented this feature in the nonrecurrent group.

(2) Another characteristic observed in all recurrent ears 
was the retro-auricular location of the keloids. Considering the complex anatomy of the ear, optimal alignment of the device was difficult to obtain in the retro-auricular area. Maintaining enough pressure in this specific site was thus challenging for the clip.

(3) As previously observed in the literature [16, 19], individuals with darker skin phenotypes tend to show a higher risk of recurrence than lighter skin patients. Similar observations were noted when comparing demographic data between the recurrent and non-recurrent groups.

\subsection{Limitations of the Study}

Despite the conduction of the study over a 10 years period, the population size was small. Indeed, the incidence of this specific diagnosis at a single health care center is low. Additionally, we believe that assessing treatment adherence would have brought more information to the literature. As showed Kadouch et al., the relation between compliance and effectiveness is very intricate in this treatment modality [16].

\section{Conclusions}

The aesthetic considerations of ear keloids are serious, especially in young patients. Excisional surgery combined with pressure therapy is an effective and well-tolerated treatment for ear keloids on a pediatric population. Previous studies on older individuals also supported the effectiveness of such a treatment protocol $[16,17,19]$. The specificity of the 3 layers pressure device lies in its advantages: high success rates on long term follow-up, transparency for skin perfusion control and adjustable pressure to prevent complications. Long term follow-up was the strength of the study and allowed for proper evaluation of treatment effectiveness. Although residual symptoms were sometimes observed, pressure therapy tends to be better tolerated than other more invasive treatments.

\section{List of Abbreviations}

ECM: extracellular matrix

HTS: hypertrophic scar

TGF- $\beta$ : Transforming Growth Factor $\beta$

\section{References}

[1] Sorg H, Tilkorn DJ, Hager S, Hauser J, Mirastschijski U. Skin Wound Healing: An Update on the Current Knowledge and Concepts. Eur Surg Res. 2017; 58 (1-2): 81-94.

[2] Berman B, Maderal A, Raphael B. Keloids and Hypertrophic Scars: Pathophysiology, Classification, and Treatment. Dermatol Surg. 2017 Jan; 43: S3-S18.

[3] Lee SS, Yosipovitch G, Chan YH, Goh CL. Pruritus, pain, and small nerve fiber function in keloids: A controlled study. J Am Acad Dermatol. 2004 Dec; 51 (6): 1002-6.

[4] Bock O, Schmid-Ott G, Malewski P, Mrowietz U. Quality of life of patients with keloid and hypertrophic scarring. Arch Dermatol Res. 2006 Apr; 297 (10): 433-8.
[5] Lee JY, Yang CC, Chao SC, Wong TW. Histopathological differential diagnosis of keloid and hypertrophic scar. Am J Dermatopathol. 2004 Oct; 26 (5): 379-84.

[6] Andrews JP, Marttala J, Macarak E, Rosenbloom J, Uitto J. Keloids: The paradigm of skin fibrosis - pathomechanisms and treatment. Matrix Biol. 2016 Apr; 51: 37-46.

[7] Alhady SM, Sivanantharajah K. Keloids in various races. A review of 175 cases. Plast Reconstr Surg. 1969 Dec; 44 (6): 564-6.

[8] Bayat A, Arscott G, Ollier WE, Grouther DA, Ferguson MW. Keloid disease: clinical relevance of single versus multiple site scars. Br J Plast Surg. 2005 Jan; 58 (1): 28-37.

[9] Butler PD, Longaker MT, Yang GP. Current Progress in Keloid Research and Treatment. J Am Coll Surg. 2008 Apr; 206 (4): 731-41.

[10] Aggarwal A, Ravikumar BC, Vinay KN, Raghukumar S, Yashovardhana DP. A comparative study of various modalities in the treatment of keloids. Int J Dermatol. 2018 Oct; 57 (10): 1192-1200.

[11] Sunaga A, Kamochi H, Sarukawa S, Uda H, Sugawara Y, Asahi R, et al. Reconstitution of Human Keloids in Mouse Skin. Plast Reconstr Surg Glob Open. 2017 Apr; 5 (4): 1304.

[12] Coentro JQ, Pugliese E, Hanley G, Raghunath M, Zeugolis DI. Current and upcoming therapies to modulate skin scarring and fibrosis. Adv Drug Deliv Rev [Internet]. 2018 Aug 30 [cited 2019 Feb 23]; Available from: http://www.sciencedirect.com/science/article/pii/S0169409X1 8302072 .

[13] Niessen FB, Spauwen P, Schalkwijk J, Kon M. On the Nature of Hypertrophic Scars and Keloids: A Review. Plast Reconstr Surg. 1999 Oct 1; 104 (5): 1435-58.

[14] Rosen DJ, Patel MK, Freeman K, Weiss PR. A Primary Protocol for the Management of Ear Keloids: Results of Excision Combined with Intraoperative and Postoperative Steroid Injections. Plast Reconstr Surg. 2007 Oct; 120 (5): $1395-400$.

[15] Ogawa R, Yoshitatsu S, Yoshida K, Miyashita T. Is radiation therapy for keloids acceptable? The risk of radiation-induced carcinogenesis. Plast Reconstr Surg. 2009 Oct; 124 (4): 1196201.

[16] Kadouch DJ, van der Veer WM, Mahdavian Delavary B, Kerkdijk D, Niessen FB. Therapeutic hotline: an alternative adjuvant treatment after ear keloid excision using a custommade methyl methacrylate stent. Dermatol Ther. 2010 NovDec; 23 (6): 686-92.

[17] Park TH, Seo SW, Kim JK, Chang CH. Outcomes of surgical excision with pressure therapy using magnets and identification of risk factors for recurrent keloids. Plast Reconstr Surg. 2011 Aug; 128 (2): 431-9.

[18] Nason LH. Keloids and their treatment. New Engl J Med. 1942; 226: 883-886.

[19] Tanaydin V, Beugels J, Piatkowski A, Colla C, van den Kerckhove E, Hugenholtz GCG, et al. Efficacy of custommade pressure clips for ear keloid treatment after surgical excision. J Plast Reconstr Aesthet Surg. 2016 Jan; 69 (1): $115-21$. 
[20] Lumenta DB, Siepmann E, Kamolz LP. Internet-based survey on current practice for evaluation, prevention, and treatment of scars, hypertrophic scars, and keloids. Wound Repair Regen. 2014 Jul-Aug; 22 (4): 483-91.

[21] Gauglitz GG. Management of keloids and hypertrophic scars: current and emerging options. Clin Cosmet Investig Dermatol. 2013 Apr 24; 6: 103-14.

[22] Pare A, Johnson T, Spiegel AV. The works of that famous chirurgeon Ambrose Parey translated out of Latin and compared with the French. London: EEBO Editions, ProQuest; 2011. $816 \mathrm{p}$.

[23] Gauglitz GG, Korting HC, Pavicic T, Ruzicka T, Jeschke MG. Hypertrophic scarring and keloids: pathomechanisms and current and emerging treatment strategies. Mol Med. 2011 Jan-Feb; 17 (1-2): 113-25.

[24] Savion Y, Sela M, Sharon-Buller A. Pressure earring as an adjunct to surgical removal of earlobe keloids. Dermatol Surg. 2009 Mar; 35 (3): 490-2.

[25] Mustoe TA. Evolution of silicone therapy and mechanism of action in scar management. Aesth Plast Surg. 2008 Jan; 32 (1): 82-92.

[26] Choi J, Lee EH, Park SW, Chang H. Regulation of Transforming Growth Factor $\beta 1$, Platelet-Derived Growth Factor, and Basic Fibroblast Growth Factor by Silicone Gel Sheeting in Early-Stage Scarring. Arch Plast Surg. 2015 Jan; 42 (1): 20-7.

[27] Bran GM, Brom J, Hörmann K, Stuck BA. Auricular keloids: combined therapy with a new pressure device. Arch Facial Plast Surg. 2012 Jan-Feb; 14 (1): 20-6.

[28] Chalian VA, Bennett JE, Sayoc AM, Ghalichebaf M. Auricular compression stent. J Prosthet Dent. 1985 Oct; 54 (4): 560-3. 